\title{
Smart eye using Ultrasonic sensor in Electrical vehicles for Differently Able.
}

\author{
H.Goutham ${ }^{1}$, M.A.Arun Shrivasan ${ }^{2}$, K.Arun Kumar $^{3}$,R.K.Praveen ${ }^{4}$ \\ Department of Electronics and Instrumentation, Sri Sai Ram Engineering College, Chennai-600044
}

\begin{abstract}
In this competitive world, electrical vehicles play a vital role. The cost of fuel is rising day by day. In order to meet this issue we need a vehicle that can overcome these problems. Physically challenged people prefer electric vehicles which are more convenient and reliable for them to travel. Controlling the speed in electric vehicles has been a major drawback, which needs to be rectified. The proposed system uses ultra-sonic sensors and controls the speed of the vehicle automatically. The ultrasonic sensors measure the distance of the obstacle and according to the corresponding distance the speed of the vehicle is regulated and reduced gradually. All these processes are carried out by automatic acceleration and deceleration without the guidance of the person driving the vehicle. The process of acceleration and deceleration is more important for controlling the speed of the electric vehicle. The speed is controlled proportional to the distance of the obstacle from the vehicle. Thus it can safely decelerate the speed and turn away from the obstacles. This system ensures safety while driving for the physically challenged person. In addition to the features of ultrasonic sensor, an infrared sensor is also used to avoid collision with obstacles. The variations in the pulse width with respect to changes in the speed is inferred using simulation of Proteus software.
\end{abstract}

Keywords: ultra-sonic sensor, infrared sensor, smart eye.

\subsection{An Overview of Existing System:}

\section{Introduction}

One of the greatest threats nowadays is not at the workplace, but rather on the road. Every human has to be cautious while driving on the road, to make a safe journey. According to the World Health Organization, road traffic caused an estimated 1.24 million deaths worldwide in the year 2010, down from 1.26 million in 2000. Half of all road traffic deaths are among pedestrians, cyclists and motorcyclists, and adults aged between 15 and 44 years account for $59 \%$ of deaths. 3 out of 4 road deaths are among men.. So if this is the case for normal human beings, then we have to consider the case for persons who are differently able. Physically challenged persons are those who suffer from partial or total loss of use of all their limbs and torso. They do not have a complete control over the vehicles that they are driving. The major challenge they face is to control the speed of these vehicles. Existing electric vehicle systems that are widely available in the market are those which have a maximum speed on a range of about $90-100 \mathrm{~km} / \mathrm{hr}$. Due to this factor, it just exacerbates the drivers of these vehicles. Controlling the speed of these vehicles can be considered a risk factor to the one driving as well as the obstacles such as other vehicles or pedestrians passing by while driving on roads. It may be sometimes be fatal also. The existing electric vehicles have technologies such as ABS (Anti-Locking Braking System), SRS Air Bags (Supplemental Restraint System Air Bags) which are used to ensure safety only after the accident has occurred..So there is a need of an effective system to control the speed of the vehicles that can be availed by the quadriplegic persons and those with disabled limbs to control the speed and prevent accidents.

\subsection{Proposed System:}

It is very important that the differently able are self reliant and apart from their physical agony they also suffer from mental agony. They feel downtrodden by their complete reliance on anyone else. Hence the speed of the electric vehicles can be controlled by our proposed system which control the speed of the vehicles in accordance with the obstacles passing by using an ultrasonic sensor, by which the collision of obstacles is prevented and the control of speed is ensured autonomously. In addition to the ultra-sonic sensors, infrared sensors are also added to ensure safety. Thus the proposed system acts as a "SMART EYE" because it acts as the third eye for differently able people and ensures their safety and safeguards them from terrible accidents. 


\section{Design Of The System:}

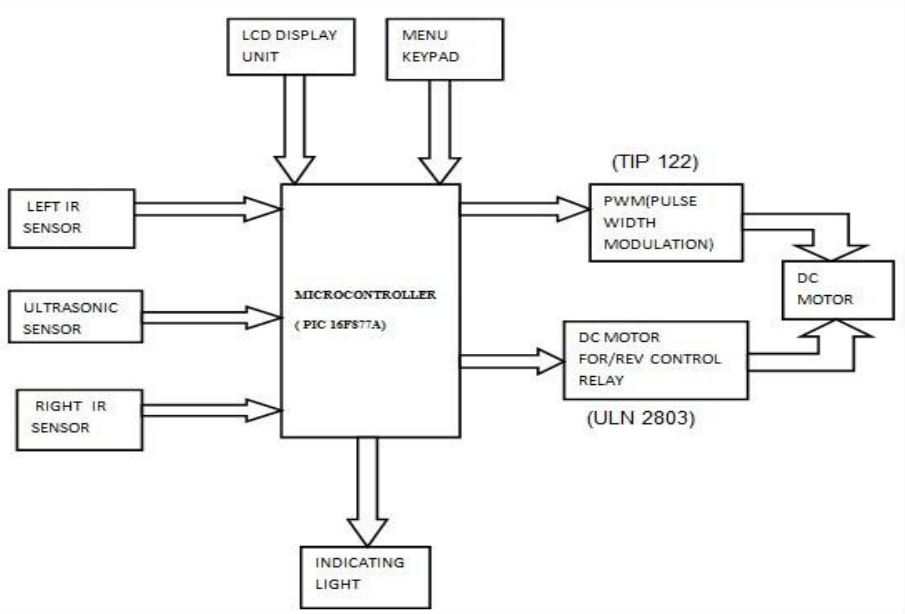

Figure 2.1: Design of the system:

\subsection{Hardware Description:}

The heart of the system is a MICROCONTROLLER

which will access the data. The microcontroller used in the system is PIC 16F877A and it is more reliable than other microcontrollers. In case of PIC16F877A the code word is written in blocks and hence acknowledgement is required only for the block of code word and hence speed of writing is more when compared to other.

To measure the distance between the electric vehicle and the obstacle an ultra-sonic sensor is used. As by the experiments, we have inferred that an ultra-sonic sensor gives more accurate output in measuring distances and its more cost effective sensor. The ultra-sonic senses the distance of the obstacle and drives the signal to the microcontroller. The ultrasonic sensor is positioned at the centre of the electric vehicle. The ultrasonic sensor used here is HC - SR04.

An additional feature is enhanced in the system, by using an infrared sensor. An infrared sensor is used in such a way that it prevents the collision of electrical vehicles during turnings and mainly used to act as a backup for the ultrasonic sensors. The infrared sensors are positioned at the left and right of the vehicle that they are aligned perfectly, so that the infrared sensors are conspicuous in sensing the obstacles. The vehicles are optimized in such a way that when the right infrared sensor detects an obstacle towards its right side, the vehicle automatically checks for any obstacle towards its left side. If the left infrared sensor gives a low output signal, i.e. there is no detection of an obstacle, and then the vehicle automatically turns towards its left side.

The sensors send the analog output signals to the $\mu \mathrm{C}$. As controller operates only on digital data, so this analog data is to be converted into digital form by using ADC. But ADC is inbuilt in PIC16F877A. Thus the analog signals are converted to digital signals and the processing of signals takes place inside the controller.

According to the signals obtained the $\mu \mathrm{C}$ sends output signals based on the pulse width modulation technique using a PWM controller. Pulse-width modulation (PWM), as it applies to motor control, is a way of delivering energy through a succession of pulses rather than a continuously variable (analog) signal. Based on the distance measured by the sensor, the PWM by increasing or decreasing pulse width, the controller regulates energy flow to the motor shaft. The motor's own inductance acts like a filter, storing energy during the "on" cycle while releasing it at a rate corresponding to the input or reference signal.

Thus the pulses are applied to the motor and the speed is gradually increased or decreased based on the distance of the obstacle. When the distance between the obstacles is too low, then the vehicle automatically stops gradually and it is indicated by a lighting system.

An LCD unit is used to display the distance of the obstacle, which is detected by the ultra-sonic sensor and the distance range is displayed on the display unit. The speed of the motor is also displayed in the LCD unit which shows the gradual increase or decrease of speed to the user driving the vehicle.

A menu keypad is included in the system which is provided for the user to switch between manual and automatic mode. The user driving the electric vehicle can give commands to control the motion of the vehicle and to start or to stop the vehicle. 


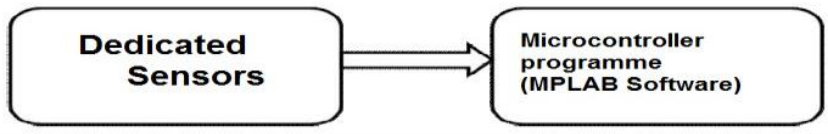

Fig. 2.2: - Block Diagram of Base Unit.

\subsection{Software Overview:}

The software selection is the backbone of the entire project. Software development includes programs written for the interfacing of the Microcontroller with LCD display, Keyboard, ADC and sensor interface. Having had a look at the software and hardware. Fundamentals of the system, the next step is to understand the software programming incorporated in the microcontroller to achieving the given task. When complete PCB with assembling is finished it comes to software where both assembly and c language is used. PIC16F877A controller is programmed for performing various operations.

The software that is used to program the code in the microcontroller is done using the software MPLAB version 8.8. The programming language used is a simple $\mathrm{C}$ language and easy to understand .CCS C (compiler software), is used to compile the program that was programmed using MPLAB software.The compiler contains Standard C operators and built-in libraries that are specific to the PIC registers. Then, using the PICKITS Programming tool, the program is downloaded from PC to the Microcontroller.

\section{Simulation Overview:}

The changes in the pulse width with respect to the distance are analyzed using software called PROTEUS (Design software). Proteus is software for microprocessor simulation, schematic capture, and printed circuit board (PCB) design.

The PCB layout of the proposed system is designed using this software and a CRO is connected to observe the change in the waveform, when the speed gradually changes with respect to changes in the distance.

When the electric vehicle is near the obstacle at distance of about less than $1 \mathrm{~m}$ then the speed decreases to zero by using pulse width modulation technique. The changes in pulse width with high peaks as shown in the digital oscilloscope using the Proteus software in the Fig.3.1.

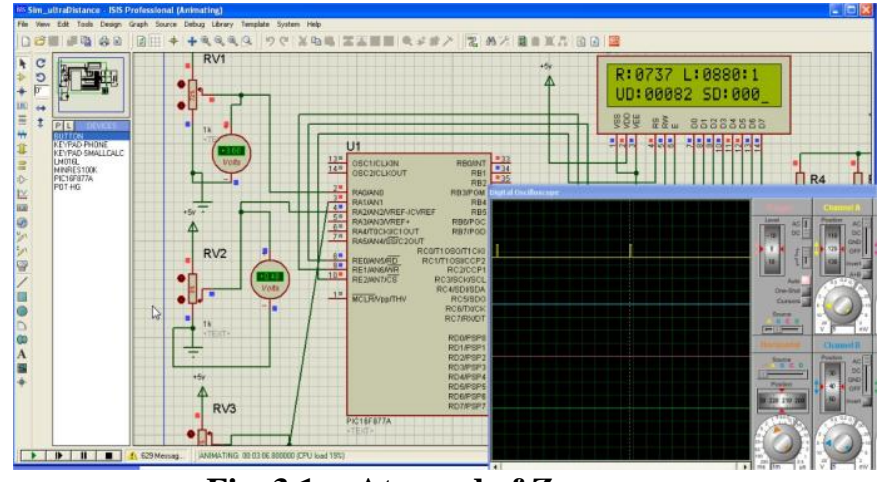

Fig. 3.1: - At speed of Zero rpm.

At a distance of about more than 10metre there is no change in the speed of the vehicle and the vehicle is at its maximum speed. Therefore, there is no change in its pulse width due to no detection in the ultrasonic sensor. This is shown in the Fig. 3.2.

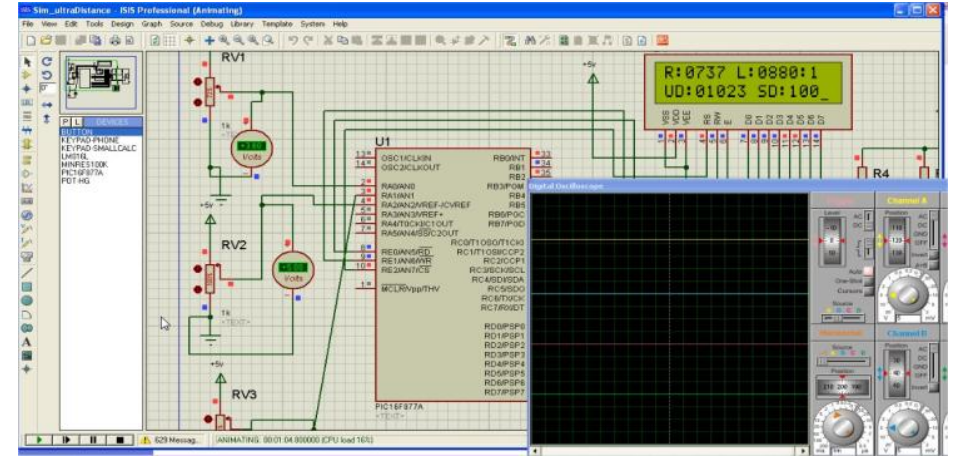

Fig. 3.2: - At speed of 100 rpm. 
When the left infrared sensor in the electric vehicle detects an obstacle towards its left side, then the right infrared sensor detects for any obstacle. If no obstacle is detected on the right side, then the electric vehicle moves towards its right side. The change in the pulse width is shown in the Fig 3.3.

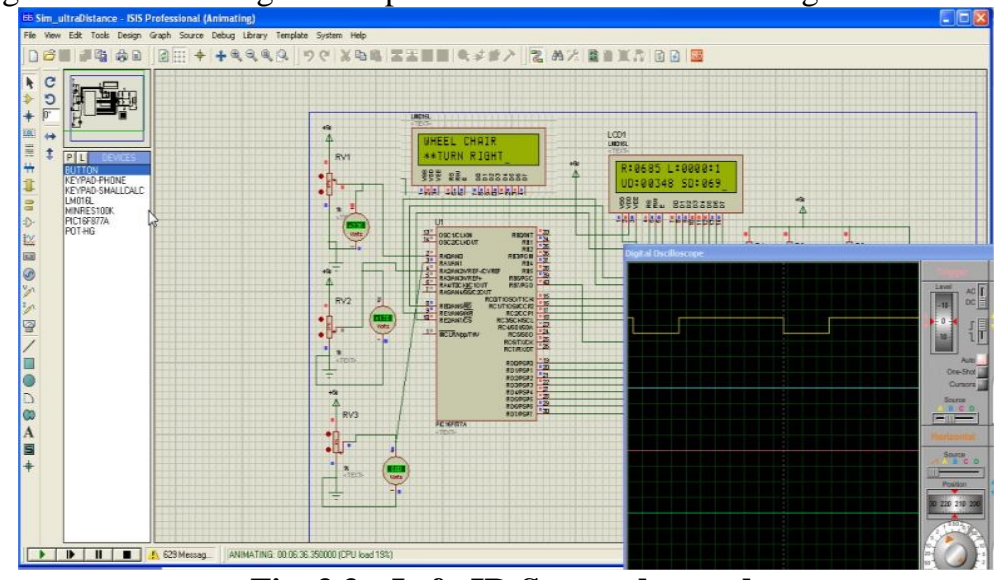

Fig. 3.3: -Left IR-Sensor detected.

When the right infrared sensor in the electric vehicle detects an obstacle towards its right side, then the left infrared sensor detects for any obstacle. If no obstacle is detected on the left side, then the electric vehicle moves towards its left side. The change in the pulse width is shown in the Fig 3.4.

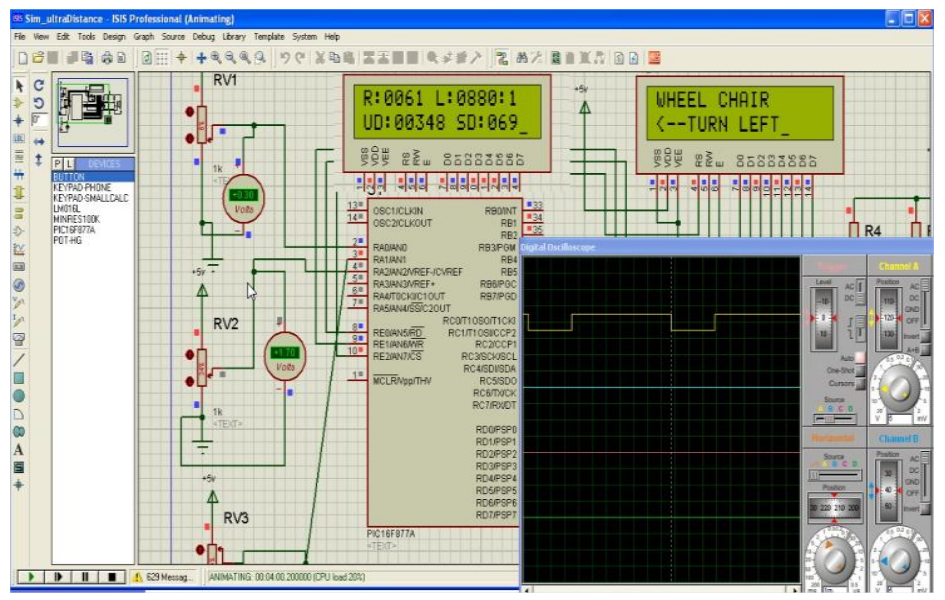

Fig. 3.4: -Right IR-Sensor detected.

\section{Benefits :}

- Low power consumption.

- Easy control for the differently able.

- $\quad$ Easy and fast to install.

- Low cost with high performance.

\section{Result:}

The prototype of a system that was proposed was successfully developed and tested in various conditions to give accurate output. The developed system proves to be an efficient and reliable system for the physically challenged people to drive safely and making it an easy control vehicle. The developed electric vehicle is shown in the Fig.5.1 and Fig.5.2. 


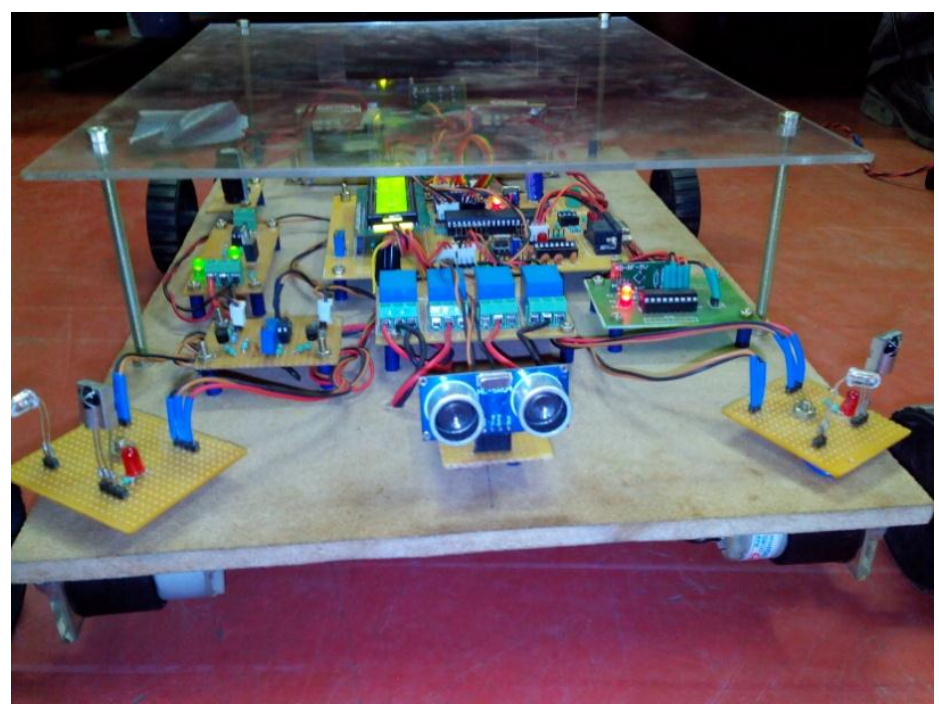

Fig.5.1 FRONT VIEW.

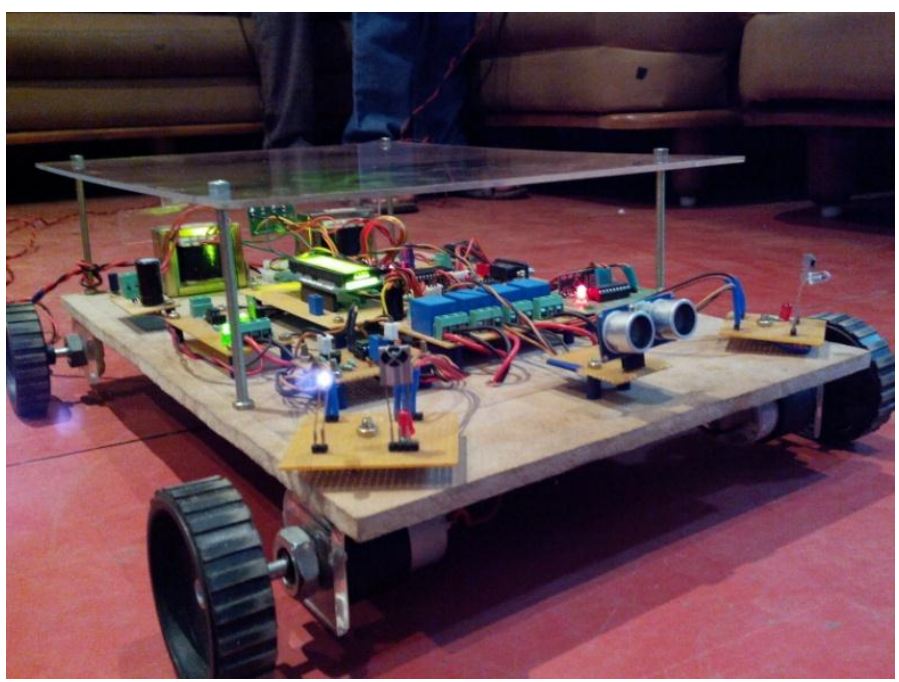

Fig.5.2 SIDEVIEW.

\section{Conclusion and Future Expansion:}

The paper discusses a speed control algorithm and obstacle avoidance algorithm used for differently able persons to control electrical vehicles. Since the system depends heavily on the ultra-sonic sensors and infrared sensors, their performance and limitation are experimentally studied and analysed as well. The ultrasonic sensors are more reliable than other sensors as it can be easily calibrated to a particular distance and can detect the objects within its range. When used for sensing functions, the ultrasonic method has unique advantages over conventional sensors:

- Discrete distances to moving objects can be detected and measured.

- Less affected by target materials and surfaces, and not affected by colour. Solid-state units have virtually unlimited, maintenance free life. Can detect small objects over long operating distances.

- Resistance to external disturbances such as vibration, infrared radiation, ambient noise, and EMI radiation.

While ultrasonic exhibit good immunity to background noise, these sensors are still likely to falsely respond to some loud noises, like the "hissing" sound produced by air hoses and relief valves.

The sensitivity and accuracy can be improved in the future by applying and improving the sensor stability by considering the external factors in the environment that affects sensor efficiency. Through research presented in this paper, we propose an intelligent electrical system for accident prevention and making the world a much better and a safe place to live. 


\section{References:}

[1]. V. Milanés, J. E. Naranjo, C. Gonzalez, J. Alonso, and T. de Pedro, "Autonomous vehicle based in cooperative gps and inertial systems," Robotic a, vol. 26, pp. 627-633, 2008.

[2]. N. B. Hounsell, B. P. Shrestha, J. Piao, and M. McDonald, "Review of urban traffic management and the impacts of new vehicle technologies,"IET Intell. Transport Syst., vol. 3, no. 4, pp. 419-428, 2009.

[3]. J. K. Kuchar and L. C. Yang, "A review of conflict detection and resolution modeling methods," IEEE Trans. Intell. Transp. Syst., vol. 1, no. 4, pp. 179-189, Dec. 2000.

[4]. Ultrasonic Ranging Module HC - SR04 http://www.micropik.com/PDF/HCSR04.pdf 